\title{
Scale Effects of the Relationships between 3D Building Morphology and Urban Heat Island: A Case Study of Provincial Capital Cities of Mainland China
}

\author{
Zhi Qiao ${ }^{1},{ }^{1}$ Xiping Han, ${ }^{1}$ Chen Wu, ${ }^{1}$ Luo Liu, ${ }^{2}$ Xinliang Xu, ${ }^{3}$ Zongyao Sun, ${ }^{4}$ \\ Wei Sun, ${ }^{5}$ Qian Cao $\mathbb{D}^{6},{ }^{6}$ and Linwan $\mathrm{Li} \mathbb{D}^{1}$ \\ ${ }^{1}$ Key Laboratory of Indoor Air Environment Quality Control, School of Environmental Science and Engineering, \\ Tianjin University, Tianjin 300350, China \\ ${ }^{2}$ Guangdong Province Key Laboratory for Land Use and Consolidation, South China Agricultural University, \\ Guangzhou 510642, China \\ ${ }^{3}$ State Key Laboratory of Resources and Environmental Information Systems, \\ Institute of Geographical Sciences and Natural Resources Research, Chinese Academy of Sciences, Beijing 100101, China \\ ${ }^{4}$ School of Architecture, Tianjin University, Tianjin 300272, China \\ ${ }^{5}$ Nanjing Institute of Geography and Limnology, Key Laboratory of Watershed Geographic Sciences, Chinese Academy of Sciences, \\ Nanjing 210008, China \\ ${ }^{6}$ School of Life Sciences, Shandong University, Qingdao 266237, China
}

Correspondence should be addressed to Qian Cao; qian.cao@sdu.edu.cn and Linwan Li; lilinwan98@163.com

Received 26 April 2020; Revised 13 October 2020; Accepted 10 November 2020; Published 27 November 2020

Academic Editor: Wen-Ze Yue

Copyright (c) 2020 Zhi Qiao et al. This is an open access article distributed under the Creative Commons Attribution License, which permits unrestricted use, distribution, and reproduction in any medium, provided the original work is properly cited.

In the process of rapid urbanization, urban heat island (UHI) effect has been showing more and more significant impacts on human well-being. Therefore, a more detailed understanding of the impact of three-dimensional (3D) building morphology on UHI effect across a continuum of spatial scales will be necessary to guide and improve the human settlement. This study selected 31 provincial capital cities of mainland China to analyze the impacts of the 3D building morphology, including the number, area, height, volume, and the surface area of the buildings, on the land surface temperature (LST). By exploring how the influence of 3D building morphology on LST changes with the increase of spatial scale (between 0 and $600 \mathrm{~m}$ radii), this study finally recognized which 3D building morphology index is the most significant index affecting LST in different cities, and which spatial scale these 3D building morphology indexes have the most significant impact on LST. The results showed that the building area is the most important 3D building morphology parameter affecting the LST, while the building height has the slightest influence on the LST. These effects are more significant in the spatial scale of $150 \mathrm{~m}-540 \mathrm{~m}$, and the spatial scale increases with the increase of building areas in developed cities. These results highlight the necessity of considering fine-grained management in the governance and alleviating of the urban thermal environment through urban planning and urban renewal strategies.

\section{Introduction}

Urbanization is a popular trend in the development of all countries in the world and a symbol of human civilization and progress [1]. The urban heat island (UHI) effect, as the most representative urban climate problem accompanied by the urbanization, is the phenomenon that the land surface temperature (LST) in urban areas is higher than that in surrounding suburbs areas [2-6]. The UHI effect could have serious consequences for urban ecological environment, such as vegetation phenology, water, and air quality $[7,8]$. These anomalies will significantly affect human health and sustainable development, which is reflected in increased morbidity and mortality [9], energy consumption [10], and even violent crimes [11]. Especially in the context of global warming and rapid urbanization, the superposition effect of 
UHI and extreme heat wave will be even more dangerous [6]. Therefore, how to alleviate the UHI effect is of great significance to improve the human settlement environment.

The UHI effect has been widely observed in the world $[3,4,6,12]$. In the framework of energy balance theory, that is, the UHI effect is caused by the changes of land surface energy balance after the artificial land surface replaces the natural surface [13]. The scholars explored the spatial (from local scale to global scale) and temporal (daily, seasonal, and interannual) changes and the driving mechanism of the UHI effect from three aspects (land use/cover change (LUCC), urban site characteristics (USCs), and landscape composition and configuration) [14-18]. In the previous stage, more studies focused on the influence of the urban two-dimensional (2D) landscape pattern on the UHI effect, while less attention on the influence of the three-dimensional (3D) building morphology $[19,20]$. Although the relative importance of $2 \mathrm{D}$ landscape pattern and $3 \mathrm{D}$ building morphology on the LST is still inconclusive, some studies have shown that the 3D building morphology may have a more complicated impact on urban micrometeorology and climate $[19,20]$. In the future, due to the contradiction between the rapid increase of urban population and the shortage of the urban construction land, while the urban construction land will expand rapidly in the horizontal direction, the urban buildings will continue to extend in the vertical direction. Therefore, it is important to reveal the spatiotemporal process and the mechanism of the urban thermal environment affected by the 3D building morphology.

The expansion of urban buildings and transportation in the vertical direction affects the local energy balance and further changes the urban internal thermal environment [21]. Therefore, the quantification of the 3D building morphology is the basis and the premise of exploring the urban thermal environment effects. In addition to the height and volume of the building, many scholars have developed numerous 3D building morphology parameters, including the frontal area index (FAI), floor area ratio (FAR), sky view factor (SVF), and height-to-width ratio $(\mathrm{H} / \mathrm{W})$, to reveal the influence of $3 \mathrm{D}$ building morphology parameters on the UHI effect $[15,22-26]$. Statistical analysis methods, including correlation analysis and multiple regression analysis, were usually used to analyze the influence of 3D building morphology on LST. Obviously, the strong interaction and collinearity between the selected 3D building morphology parameters would directly affect the accuracy of the analysis results [6]. In addition, it is difficult to compare and analyze the results of cities or regions on the different climate background conditions and development levels, and there may even be contrary conclusions. The classification method that is to classify the study area according to the building height and other parameters was used to compare the LST of different building types or analyze the correlations between the 3D building morphology parameters and LST in various areas, which can reduce the uncertainty of statistical analysis method. The most representative scheme, local climate zone (LCZ), is to divide the urban underlying surface into 17 basic types (10 building types and 7 natural types) based on the refined land classifications, land surface structures, building surface materials, and human activities [27], and it is recommended to define the UHI intensity by the LST difference among the different types of LCZ, so as to determine the theoretical basis for accurate comparison in the UHI intensity at the regional or even global scale [28-30]. However, due to the strong spatial dependence between the 3D building morphology and the LST, the spatial scale effect of the analysis variables would further reduce the accuracy of the results. The uncertainty of spatial scale effects is also reflected in spatial autocorrelation and proximity effects. This also explains the differences of UHI in the same type of LCZ. Understanding of the magnitude and scale at which urban building affects the LST in cities is hampered by the paucity of data along continuous gradients and for combinations of high spatial resolution building data and LST data [31]. Therefore, studies that quantify the effects of urban 3D building morphology on the LST across the range of spatial scales (regional, national, and even global scales) that incorporate this heterogeneity can provide a strong foundation for developing urban climate adaptation strategies. This study compares the correlations between multiple 3D building morphology parameters and the UHI intensity at the continuous spatial scale of 30-meter interval in 31 provincial capital cities of mainland China. This study will explore (1) how does variability in the multiple 3D building morphology parameters affect the UHI intensity? (2) How do these effects vary with the spatial scale at which high spatial resolution building data and LST data are analyzed? The conclusions may be of great practical significance for regulating the urban thermal environment through urban planning and urban renewal [32].

\section{Data and Methodology}

2.1. Study Area. In this study, 31 provincial capital cities in mainland China were taken as the research area (Figure 1). As the most important political city in each provincial administrative region, these provincial capital cities are representative in the economic level and city scale. In addition, these selected cities are widely distributed in different climate zones, which is convenient for the analysis of regional differences.

2.2. Data Source. In this paper, Landsat- 8 data, developed by the National Aeronautics and Space Administration (NASA) and United States Geological Survey (USGS), were collected for the LST inversion. In order to be consistent with the collection time of urban building data, the selection principle of Landsat data is the summer image covering each provincial capital city with no (or less) cloud cover and ideal atmospheric visibility. Since some cities have always been affected by clouds, the time span of the collected remote sensing images was extended from 2014 to 2018 (Supplementary Materials Table 1).

The building vector data were obtained from the Resource and Environment Data Center, Chinese Academy of Sciences (http://www.resdc.cn/Default.aspx). The dataset contains the attributes of urban building area and height, which are used to calculate the urban 3D building morphology parameters. 


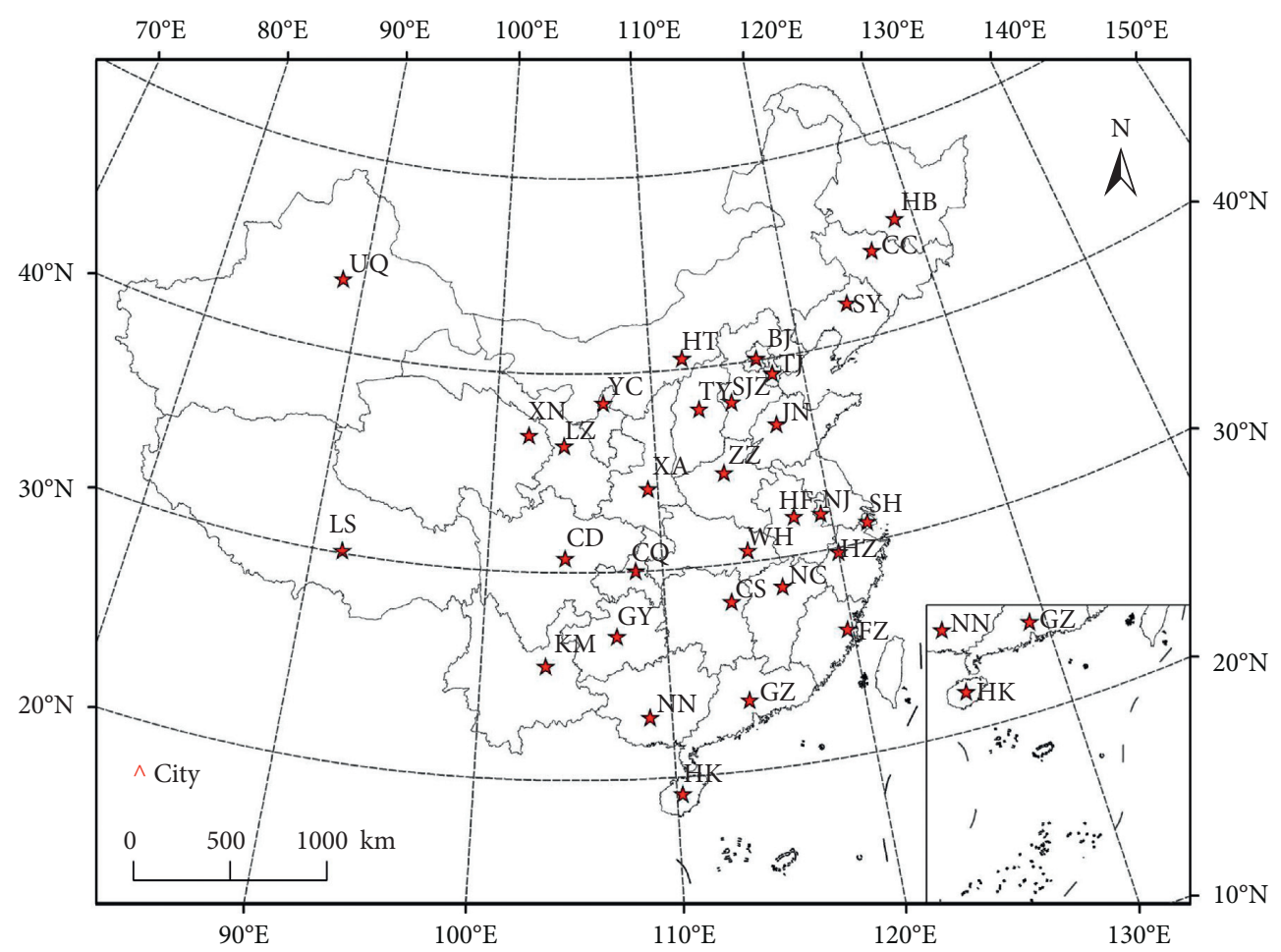

Figure 1: The locations of 31 provincial capital cities in mainland China. Note: Beijing (BJ), Changchun (CC), Changsha (CS), Chengdu (CD), Chongqing (CQ), Fuzhou (FZ), Guangzhou (GZ), Guiyang (GY), Haikou (HK), Hangzhou (HZ), Harbin (HB), Hefei (HF), Hohhot (HT), Jinan (JN), Kunming (KM), Lanzhou (LZ), Lhasa (LS), Nanchang (NC), Nanjing (NJ), Nanning (NN), Shanghai (SH), Shenyang (SY), Shijiazhuang (SJZ), Taiyuan (TY), Tianjin (TJ), Urumqi (UQ), Wuhan (WH), Xi' an (XA) and Xining (XN), Yinchuan (YC), and Zhengzhou (ZZ).

\section{Methods}

3.1. The LST Inversion. In this study, the LST of Landsat-8 was retrieved via a single-channel method. The calculation was divided into the following three procedures.

3.1.1. Calculating the Brightness Temperatures. The brightness temperature was calculated based on the Landsat- 8 data user's handbook as follows [33]:

$$
\begin{aligned}
& L_{\lambda}=\text { gains } \times \mathrm{DN}+\text { biases }, \\
& T=\frac{K_{2}}{\ln \left(\left(K_{1} / L_{\lambda}\right)+1\right)},
\end{aligned}
$$

where $L_{\lambda}$ is the radiation intensity received by the Thematic Mapper (TM). Gains is the gain factor (unit: $\left.\left(\mathrm{W} \cdot \mathrm{m}^{-2} \cdot \mathrm{sr}^{-1} \mu \mathrm{m}^{-1}\right) / \mathrm{DN}\right)$ ) and $\mathrm{DN}$ is the digital number. Biases is the offset coefficient (unit: $\mathrm{W} \cdot \mathrm{m}^{-2} \cdot \mathrm{sr}^{-1} \mu \mathrm{m}^{-1}$ ). $T$ is the brightness temperatures (unit: K) and $K_{1}$ and $K_{2}$ are constants. $L_{\lambda}$ is the luminance value of the unit spectral range (unit: $\mathrm{mW} \cdot \mathrm{cm}^{-2} \cdot \mathrm{sr}^{-1} \mu \mathrm{m}^{-1}$ ). The values of $K_{1}$ and $K_{2}$ of band 10 of Landsat- 8 TIRS are $774.89 \mathrm{~W} \cdot \mathrm{m}^{-2} \cdot \mathrm{sr}^{-1} \mu \mathrm{m}^{-1}$ and $1321.08 \mathrm{~K}$.
3.1.2. Calculating the Specific Emissivity ( $)$ ). The specific emissivity is one of the most important parameters for retrieving the LST, which represents the ability of the object to emit electromagnetic radiation. In this study, the classical empirical formula proposed by Van de Griend in 1993 was used to calculate the specific emissivity [34]:

$$
\varepsilon=1.009+0.047 \ln (\mathrm{NDVI}),
$$

where NDVI is the normalized vegetation index [35], NDVI $>0$ or $\varepsilon=0$.

3.1.3. Retrieving the LST. The single-window algorithm model is as follows [36]:

$$
T_{s}=A_{0}+A_{1} T_{10}-A_{2} T_{11}
$$

where $T_{s}$ is the LST (unit: K), $T_{10}$ and $T_{11}$ are the brightness temperatures, and $A_{0}, A_{1}$, and $A_{2}$ are coefficients determined by the atmospheric transmittance and land surface emissivity in both TIRS bands. The specific calculation process is as follows: 


$$
\begin{aligned}
& A_{0}=a_{10} E_{1}-a_{11} E_{2}, \\
& A_{1}=1+A+b_{10} E_{1}, \\
& A_{2}=A+b_{11} E_{2}, \\
& E_{1}=\frac{D_{11}\left(1-C_{10}-D_{10}\right)}{E_{0}}, \\
& E_{2}=\frac{D_{10}\left(1-C_{11}-D_{11}\right)}{E_{0}}, \\
& A=\frac{D_{10}}{E_{0}}, \\
& E_{0}=D_{11} C_{10}-D_{10} C_{11},
\end{aligned}
$$

where $C_{10}, C_{11}, D_{10}$, and $D_{11}$ are calculated as follows [37]:

$$
\begin{aligned}
& C_{i}=\varepsilon_{i} \tau_{i}(\theta), \\
& D_{i}=\left[1-\tau_{i}(\theta)\right]\left[1+\left(1-\varepsilon_{i}\right) \tau_{i}(\theta)\right],
\end{aligned}
$$

where $\varepsilon_{i}$ is the land surface emissivity of band $i$ and $\tau_{i}(\theta)$ is the atmospheric transmittance for a given zenith view angle $\theta$ in band $i$. The regression coefficients $a_{i}$ and $b_{i}$ for Landsat- 8 TIRS bands 10 and 11 at different ranges of temperatures are shown in Table 1 . The atmospheric transmittance, $\tau_{i}$, is shown in Table 2 . These parameters can be obtained from $[36,38]$.

3.1.4. The Relative UHI Intensity. Because the selected provincial capital cities are located in different terrain, climate region, and development stage, the relative UHI intensity index is used to represent the LST to ensure the relative consistency of the LST among different provincial capital cities.

The relative UHI intensity index is as follows [39]:

$$
T_{R}=\frac{\left(T_{i}-T_{a}\right)}{T_{a}},
$$

where $T_{R}$ is defined as the relative UHI intensity of a certain point $i$ in the study area, which is a dimensionless ratio. $T_{i}$ is the LST at this point $i$ and $T_{a}$ is the average LST in the study area.

3.1.5. Urban 3D Building Morphology Parameters. In this study, five building parameters, including the number $(N)$, the area $(A)$, the height $(H)$, the surface area $(S)$, and the volume $(V)$ of the buildings, were selected to represent the 3D building morphology of these provincial capital cities. Firstly, the $30 \mathrm{~m} \times 30 \mathrm{~m}$ fishnets were drawn corresponding to Landsat- 8 LST data, and then, the 3D building morphology parameters in each fishnet were counted. Among them, the number, the height, the area, and the perimeter $(L)$ of the building could be directly calculated. Finally, the surface area and volume of buildings in each fishnet could be further calculated according to these basic building parameters:

$$
\begin{aligned}
S & =L \times H+A, \\
V & =H \times A .
\end{aligned}
$$

3.1.6. The Analysis Method of Spatial Scale Effect. The kernel density function was used to characterize the $3 \mathrm{D}$ building morphology parameters and the LST characteristics. The kernel density analysis method is suitable for the spatial model in which the point elements have a significant impact on the peripheral environment, and these effects show a decreasing buffer effect.

The formula of the kernel density analysis method is as follows:

$$
D\left(x_{i}, y_{i}\right)=\frac{1}{n r} \sum_{j=1}^{n} k(d, r),
$$

where $D\left(x_{i}, y_{i}\right)$ is the kernel density value of any location $\left(x_{i}, y_{i}\right)$ in the study area and $r$ is the farthest radius that this location can affect. $n$ is the number of point features with the range $r$ around the location. The $k$ function represents the spatial weight function. Notice that this function requires that all the point features conform to a normal distribution. $d$ represents the Euclidean distance between the current feature point $j$ and location $\left(x_{i}, y_{i}\right)$.

According to the spatial resolution of LST, the minimum bandwidth (spatial scale) of kernel density function was set as 30 meters, which was increased by 30 meters to 600 meters [40]. Under the condition of different spatial scale, five correlation matrixes between the kernel densities of the $3 \mathrm{D}$ building morphology parameters and the relative UHI intensity were constructed. By comparing the correlation coefficients and significance levels of these correlation matrixes under different spatial scales, the most significant 3D morphology parameters that affect the LST and the most significant spatial scale effect of the 3D building morphology on the LST were identified.

Before constructing the correlation matrixes between the kernel densities of the $3 \mathrm{D}$ building morphology parameters and the relative UHI intensity, we noticed that these kernel densities do not fully conform to the normal distribution, so we need to logarithmically process the kernel density results of the 3D building morphology parameters and the relative UHI intensity:

$$
\mathrm{DD}=\lg (D)
$$

where $\mathrm{DD}$ is the logarithm of the kernel density value $D$ at any point $\left(x_{i}, y_{i}\right)$ in the study area.

In this study, the ArcGIS 10.2 band collection statistics tool was used to construct the correlation matrixes between the kernel densities of the 3D building morphology parameters and the relative UHI intensity in order to obtain the covariances and correlation coefficients between the $3 \mathrm{D}$ building morphology parameters and the relative UHI intensity: 
TABLE 1: Inversion regression coefficients of TIRS in different temperature ranges $\left(r_{10}^{2}\right.$ and $r_{11}^{2}$ are the determinants of the fit).

\begin{tabular}{|c|c|c|c|c|c|c|}
\hline Temperature range $\left({ }^{\circ} \mathrm{C}\right)$ & $a_{10}$ & $b_{10}$ & $r_{10}^{2}$ & $a_{11}$ & $b_{11}$ & $r_{11}^{2}$ \\
\hline $0-30$ & -59.1391 & 0.4213 & 0.9991 & -63.3921 & 0.4565 & 0.9991 \\
\hline $0-40$ & -60.9196 & 0.4276 & 0.9985 & -65.2240 & 0.4629 & 0.9985 \\
\hline $10-40$ & -62.8065 & 0.4338 & 0.9992 & -67.1728 & 0.4694 & 0.9992 \\
\hline $10-50$ & -64.6081 & 0.4399 & 0.9986 & -69.0215 & 0.4756 & 0.9986 \\
\hline
\end{tabular}

TABLE 2: Relationships between atmospheric transmittance $(\tau)$ and water vapor $(\omega)$ content when the water vapor content is from 0.5 to $3 \mathrm{~g} / \mathrm{cm}^{2}$.

\begin{tabular}{lccc}
\hline Profile & Atmospheric transmittance estimation equation & $r^{2}$ & Standard error of estimate \\
\hline \multirow{2}{*}{ 1976 US standard atmosphere model } & $\tau_{10}=-0.1146 \omega+1.0286$ & 0.9882 & 0.0094 \\
& $\tau_{11}=-0.1568 \omega+1.0083$ & 0.9947 & 0.0086 \\
\hline \multirow{2}{*}{ Midlatitude summer } & $\tau_{10}=-0.1134 \omega+1.0335$ & 0.986 & 0.0101 \\
& $\tau_{11}=-0.1546 \omega+1.0078$ & 0.996 & 0.0073 \\
\hline
\end{tabular}

$$
\begin{aligned}
& \operatorname{Cov}_{\text {DDUHII }}=\frac{\sum_{k=1}^{N}\left(\mathrm{DD}_{k}-\overline{\mathrm{DD}}\right)\left(\mathrm{UHII}_{k}-\overline{\mathrm{UHII}}\right)}{N-1}, \\
& \operatorname{Corr}_{\mathrm{DDUHI}}=\frac{\operatorname{Cov}_{\mathrm{DDUHII}}}{\delta_{\mathrm{DD}} \delta_{\mathrm{UHI}}},
\end{aligned}
$$

where $\operatorname{Cov}_{\text {DDUHII }}$ represents the covariance between the kernel densities of the 3D building morphology parameters and the kernel density of the relative UHI intensity, $\mathrm{DD}_{k}$ represents the logarithmic value of the kernel density of the 3D building morphology parameters in a certain pixel, and $\mathrm{UHII}_{k}$ is the kernel density of the relative UHI intensity of the pixel. $N$ is the total number of pixels in the study area; Corr $_{\text {DDUHII } i}$ is the correlation coefficient between the kernel densities of the 3D building morphology parameters and the kernel density of the relative UHI intensity, and $\delta_{\mathrm{DD}}$ and $\delta_{\mathrm{UHII}}$ represent the standard deviation of the logarithm of the kernel densities of the 3D building morphology parameters and the kernel density of the relative UHI intensity, respectively.

\section{Results}

4.1. The Spatial Pattern of Urban Buildings. This study focused on the areas within the outer ring road of each provincial capital city because most of the urban buildings are concentrated in these areas (Figure 2). Urban buildings were divided into three levels according to height, namely, low-rise buildings, middle-rise buildings, and high-rise buildings [27].

The height and density of urban buildings changed significantly from the periphery to the center of the city. The buildings in the peripheral area of the city were mainly lowrise buildings, which had large single area and sparse spatial distribution. With the decrease of the distance to the center of the city, the height of buildings increased significantly and the number of middle-rise buildings increased, but the areas decreased slightly, and the spatial distribution was gradually more compact; in the center of the city, high-rise buildings did not show obvious aggregation effect, usually single high- rise building was mainly inlaid in the middle-rise building cluster area.

4.2. The Spatial Pattern of Relative UHI Intensity. Compared with the UHI intensity, most of the pixels in each city were defined as the moderate temperature region, but there were obvious UHI centers and urban cold island (UCI) centers (Figure 3). For example, Changsha, Hangzhou, Nanchang, and Wuhan city had the obvious UCIs because of the large area of water bodies distributed in the center of the cities. There were three types of spatial distribution characteristics of UHI. (1) The single UHI center, such as Haikou city. This was because the central area carries most of the urban functions, which made the central area produce a strong aggregation effect. (2) The central radiant, such as Tianjin and Zhengzhou city. These high-intensity UHI patches were distributed along the (ring) roads and railway, forming UHI network and expanding to the urban periphery with urban construction land. (3) The multiple UHI centers, that is, there were many high-temperature centers in the study area. The most typical representative was Shijiazhuang city. The functions of these cities might be scattered, and different regions carried different urban functions, resulting in the distributions of urban buildings, and populations were not centralized, so it was easier to form multiple UHI centers.

4.3. Impacts of 3D Building Morphology Parameters on the Relative UHI Intensity. Obviously, the correlation coefficient between the building area and the relative UHI intensity was the largest, and the correlation coefficient between building height and the relative UHI intensity is the smallest (Figure 4). In contrast, the strong-to-weak sequence of $3 \mathrm{D}$ building morphology parameters attributed on the relative UHI intensity is as follows: building area $>$ building number $>$ building surface area $>$ building volume $>$ building height. Although the significances of these effects of the 3D building morphology parameters on the relative UHI intensity in different cities were different, the fluctuation trends are basically similar. 


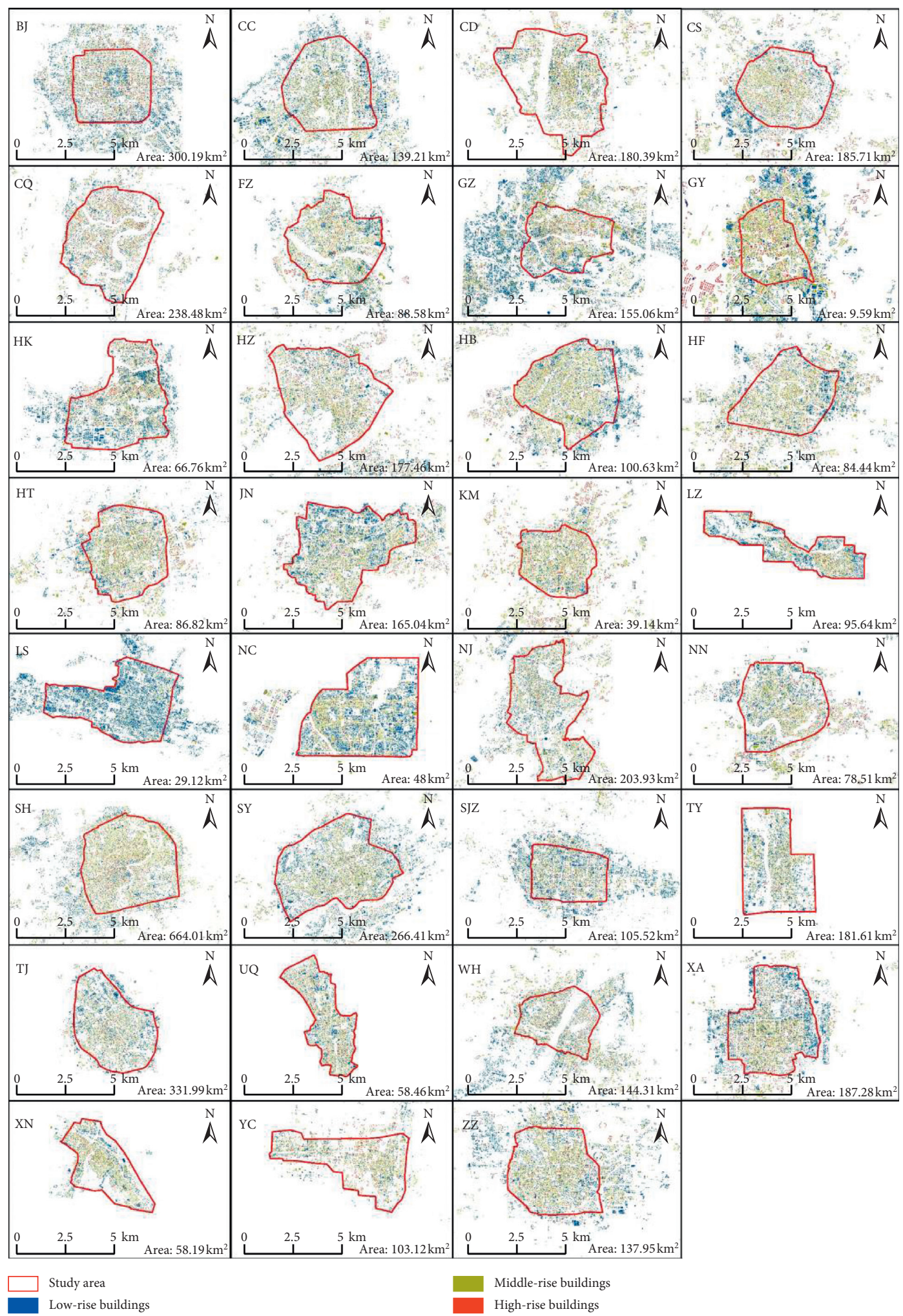

FIgURE 2: The spatial distribution of urban buildings in provincial cities of mainland China.

When the spatial scale was less than $60 \mathrm{~m}$, the low correlation coefficients between the 3D building morphology parameters and the relative UHI intensity showed that the influence of 3D building morphology on the UHI effect was extremely slight. With the increase of spatial scale from $60 \mathrm{~m}$ to $150 \mathrm{~m}$, the correlation coefficients 


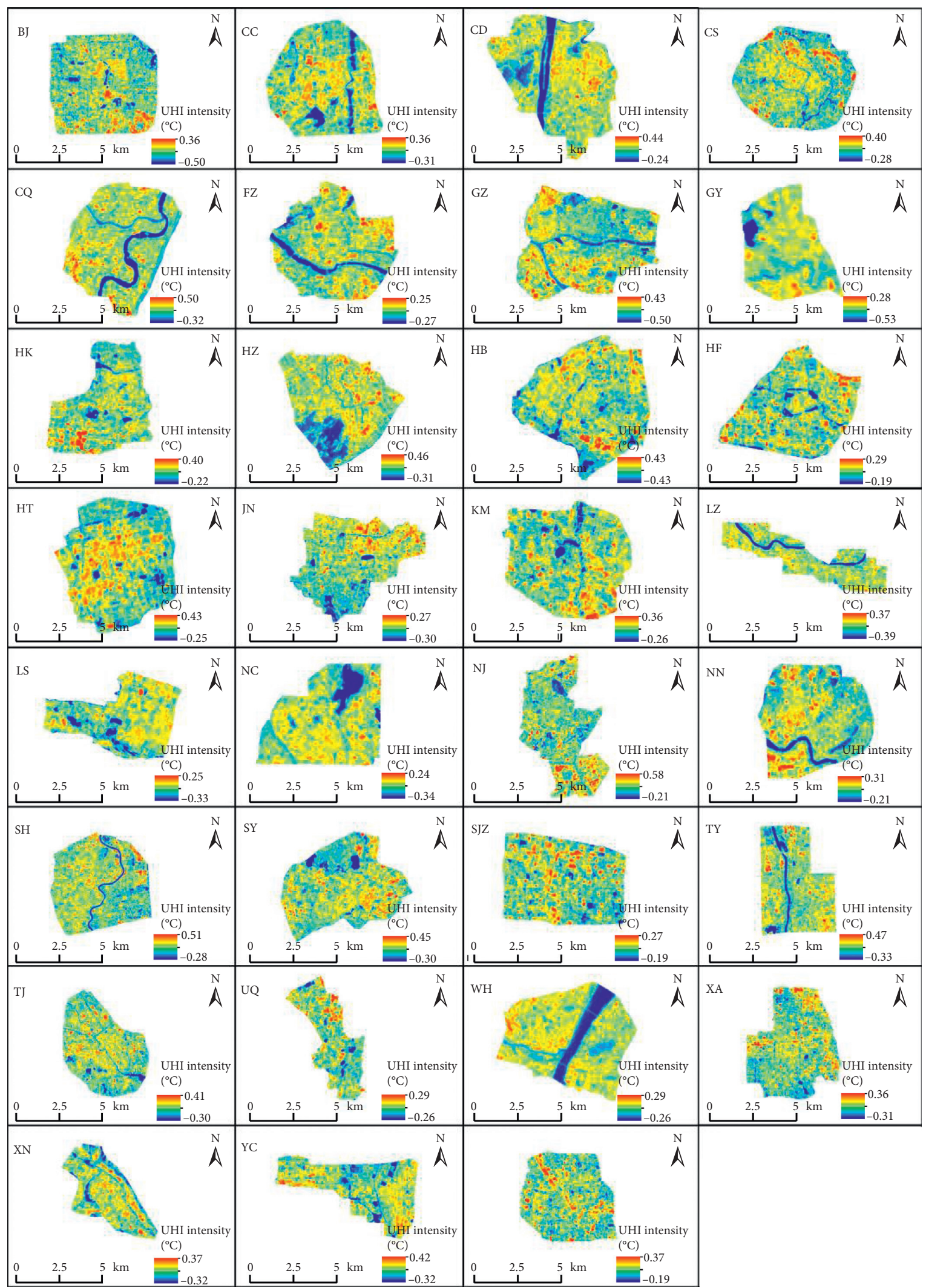

FIgURE 3: The spatial distribution of the relative UHI intensity in provincial cities of mainland China.

between $3 \mathrm{D}$ building morphology parameters and the relative UHI intensity in each provincial capital city basically increased linearly within this range, which indicated that the influence of 3D building morphology parameters on the UHI effect increased significantly within this range. 

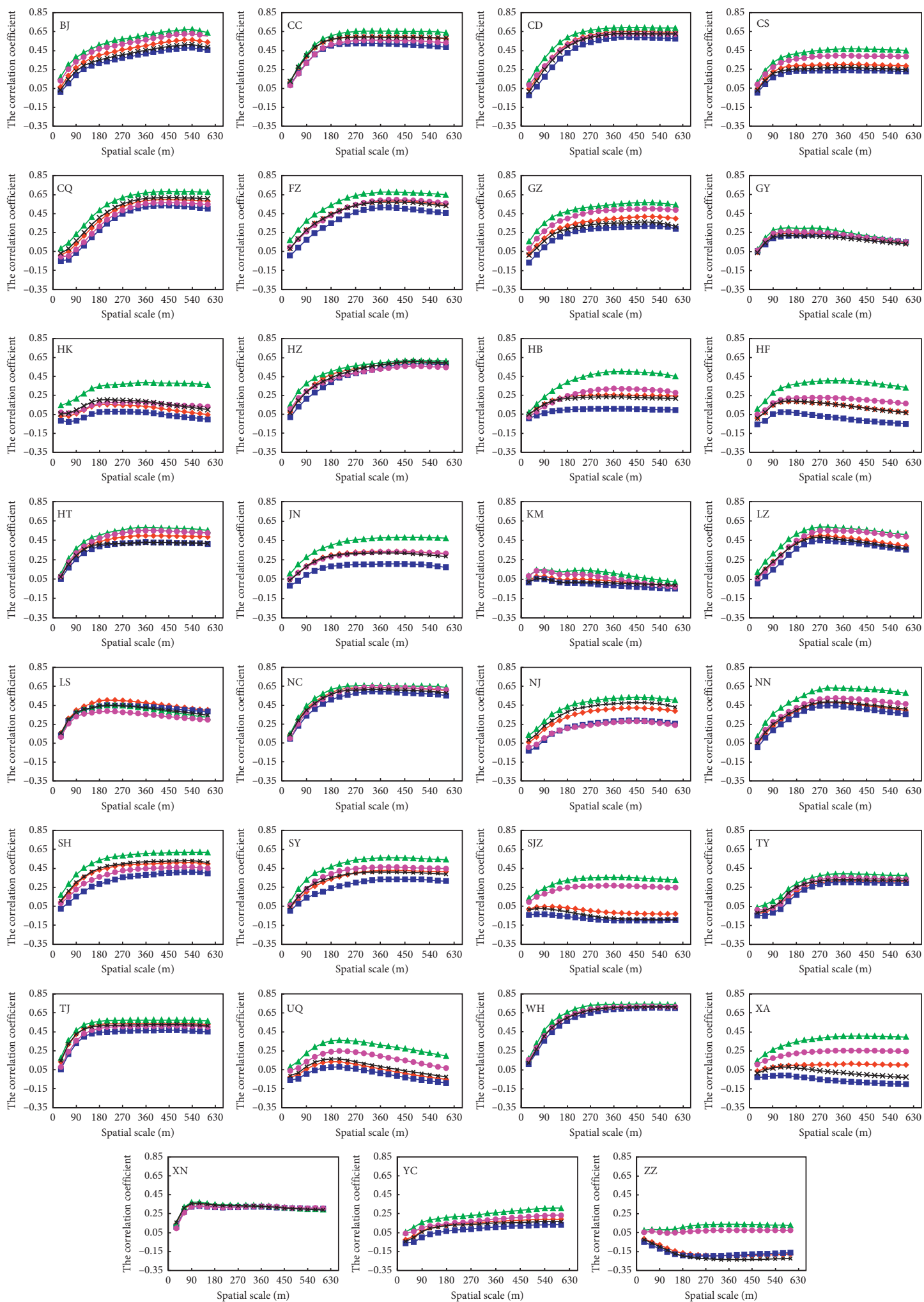

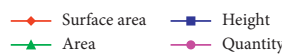

FIGURE 4: Pearson correlation coefficients between 3D building morphology parameters and the relative UHI intensity at different spatial scales. 

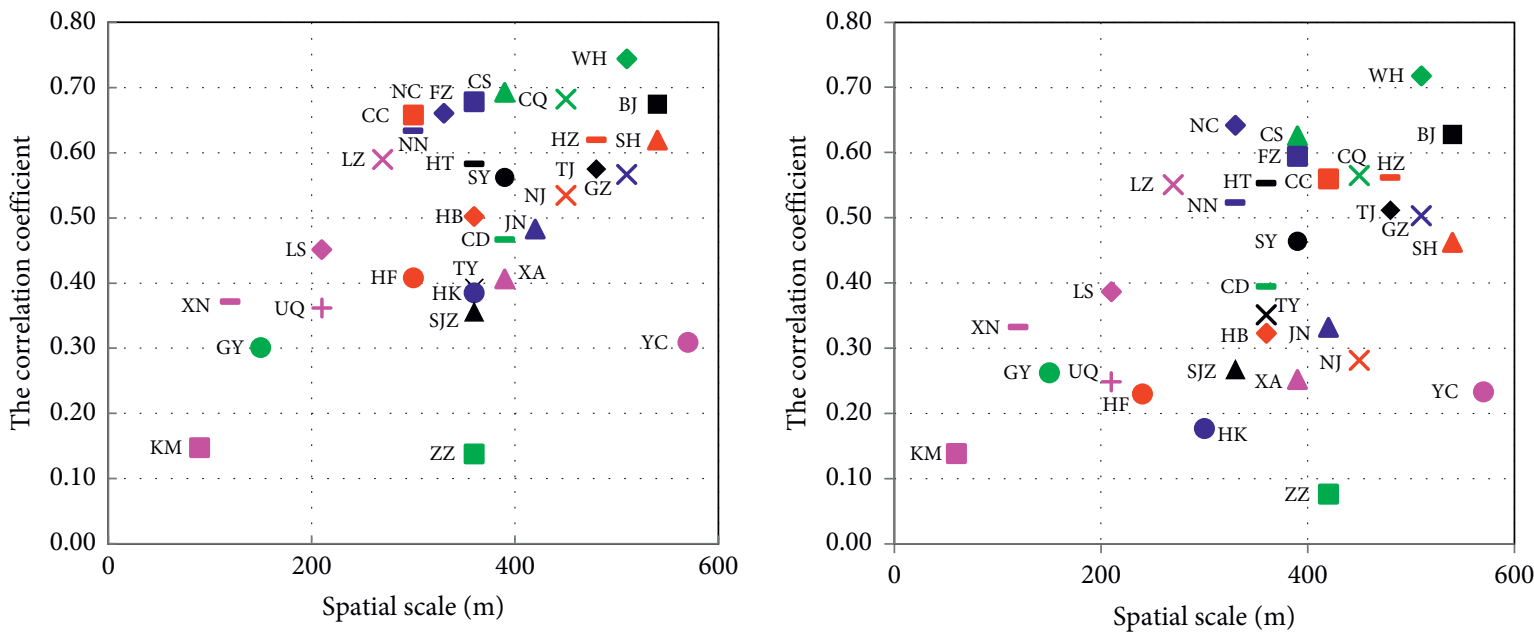

\begin{tabular}{|c|c|c|c|c|}
\hline YC & $\mathrm{HF}$ & $\triangle \mathrm{CS}$ & GY & $\times \mathrm{TY}$ \\
\hline$\diamond \mathrm{TJ}$ & $+\mathrm{UQ}$ & $\triangle \mathrm{XA}$ & $\triangle \mathrm{SH}$ & $\mathrm{ZZ}$ \\
\hline$\Delta \mathrm{JN}$ & $\square \mathrm{BJ}$ & $\mathrm{HB}$ & $-\mathrm{HZ}$ & $-\mathrm{NN}$ \\
\hline$-\mathrm{CD}$ & $\times \mathrm{GZ}$ & $\boldsymbol{\Delta} \mathrm{SJZ}$ & $\times \mathrm{NJ}$ & $\diamond \mathrm{LS}$ \\
\hline - HT & $\rightarrow \mathrm{WH}$ & $\square \mathrm{CC}$ & - SY & $-\mathrm{XN}$ \\
\hline HK & $\times C Q$ & $\times \mathrm{LZ}$ & $\square \mathrm{FZ}$ & KM \\
\hline
\end{tabular}

(a)

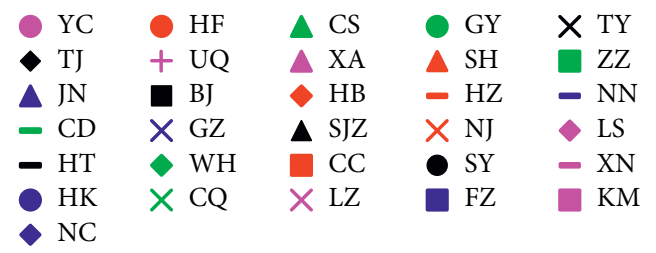

(b)
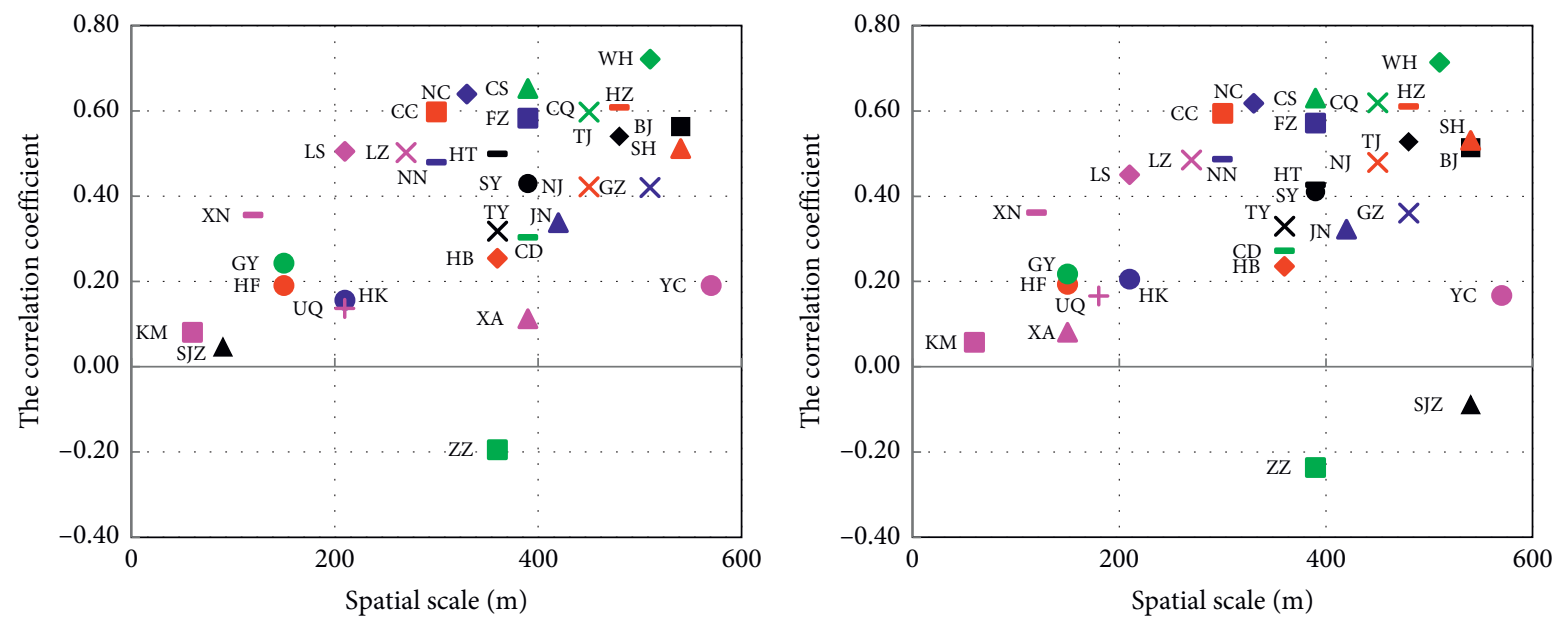

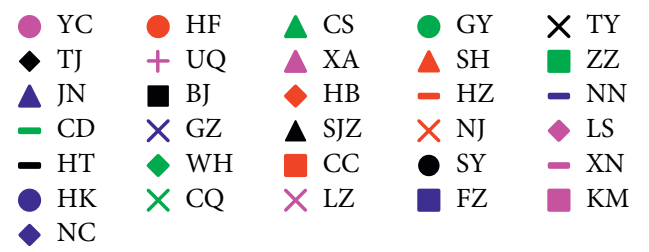

\begin{tabular}{|c|c|c|c|c|}
\hline YC & $\mathrm{HF}$ & $\triangle \mathrm{CS}$ & $\mathrm{GY}$ & $\times \mathrm{TY}$ \\
\hline TJ & + UQ & $\triangle \mathrm{XA}$ & $\triangle \mathrm{SH}$ & $\mathrm{ZZ}$ \\
\hline JN & 口 BJ & $\longrightarrow \mathrm{HB}$ & $-\mathrm{HZ}$ & - NN \\
\hline CD & $\times \mathrm{GZ}$ & $\boldsymbol{\Delta} \mathrm{SJZ}$ & $\times \mathrm{NJ}$ & LS \\
\hline HT & $\longrightarrow \mathrm{WH}$ & $\square \mathrm{CC}$ & - SY & XN \\
\hline & $\times C Q$ & $\times \mathrm{LZ}$ & FZ & KM \\
\hline
\end{tabular}

(c)

(d)

FIgURE 5: Continued. 


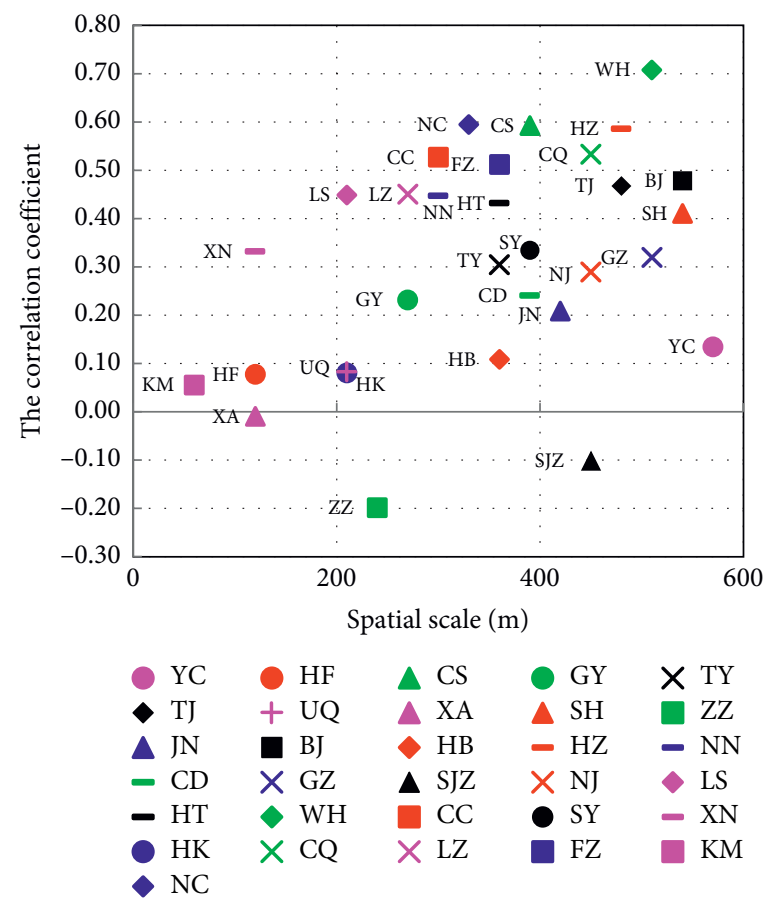

(e)

FIGURE 5: The threshold of spatial scale under the maximum correlation coefficient between the 3D building morphology parameters and the relative UHI intensity. (a) Area; (b) quantity; (c) surface area; (d) volume; (e) height.

When the spatial scale exceeded $150 \mathrm{~m}$, there were three cases in the correlation coefficient between the 3D building morphology parameters and the relative UHI intensity. (1) The correlation coefficients between all 3D building morphology parameters and the UHI intensity continued to increase, but the increase rate became slower, and finally showed a slight decline or remained unchanged. The increasing trend of these correlations could reach up to $540 \mathrm{~m}$, such as Beijing and Shanghai. (2) When the correlation coefficients between all 3D building morphology parameters and the UHI intensity reached the maximum value, the correlation coefficients decreased with the increase of spatial scale. This situation was represented by Guiyang city, Hefei city, Lanzhou city, Lhasa city, Nanning city, Urumqi city, and Xining city. In these cities, when the correlation coefficient reached the maximum, the spatial scale was between $150 \mathrm{~m}$ and $300 \mathrm{~m}$. (3) The influences of different 3D building morphology parameters on the UHI intensity varied with the increase of spatial scale. Shijiazhuang and Zhengzhou city were the most typical cities. In Shijiazhuang city, when the spatial scale reached 150 meters, the influences of the surface area, volume, and height of the building on the UHI intensity continued to decrease. When the spatial scale reached 180 meters, these building parameters even had negative impacts on the relative UHI intensity. Similarly, the negative influences of the surface area, volume, and height of the building on the UHI intensity increased in Zhengzhou city, while the influences of the area and quantity on the relative UHI intensity remained stable. The influence of building area, quantity, and the surface area on the relative UHI intensity in Xi' an city was opposite to that of the volume and the height of the building.

We further showed the thresholds of the spatial scale when $3 \mathrm{D}$ building morphology parameters are most related to the relative UHI intensity (Figure 5). When the correlations between $3 \mathrm{D}$ building morphology parameters and the relative UHI intensity were most significant, the thresholds of spatial scale for various 3D building morphology parameters were generally consistent in these provincial capital cities. Building area, as the most relevant factor of the UHI intensity, had positive effects in all cities. In $80.65 \%$ of cities, the thresholds of spatial scale were between $300 \mathrm{~m}$ and $540 \mathrm{~m}$. Obviously, in the international metropolitan areas such as Beijing, Shanghai, Wuhan, Chongqing, Hangzhou, Tianjin, Guangzhou, and Nanjing city, these spatial scale thresholds were larger and the correlation coefficients were greater than 0.5. However, in these cities, including Kunming, Guiyang, Xining, Urumqi, and Lhasa, with a superior ecological environment, the thresholds of the spatial scale were less than $210 \mathrm{~m}$ when the building area had the most significant impact on the relative UHI intensity. For other 3D building morphology parameters, when the correlations between these parameters and the relative UHI intensity reached the maximum values, the thresholds of spatial scale among cities were basically similar. 


\section{Discussion}

Because the influences of urban buildings on LST are constantly changing within a certain buffer zone, this study used the kernel density analysis method to analyze the effects of five 3D building morphology parameters on the relative UHI intensity under the continuous spatial scale of 30-meter interval by setting different bandwidths. When the spatial scale (bandwidth) is small, the spatial distributions of kernel density focus more on the microclimate relationship between individual buildings and adjacent buildings. These urban microclimate processes may be more complex, resulting in no obvious regularity, so the correlations between building morphology parameters and the relative UHI intensity are weak. When the spatial scale increases, the spatial distributions of kernel density gradually present the overall characteristics of the architectural groups or urban functional areas on urban thermal environment. It is of great significance to explore the most suitable threshold for adapting to and mitigating urban climate change through urban renewal and urban planning.

However, the underlying surface of the city is more complex; in addition to the buildings, there are open spaces (such as squares, roads, water bodies, and green spaces). In this study, only the influence of the buildings on LST was considered, so the results of kernel density analysis would be overestimated or underestimated. When the spatial scale (bandwidth) is set too small or too large, the climate effects caused by these neglected open spaces will affect the accuracy of the results and reduce the correlation between 3D building morphology parameters and the relative UHI intensity. In practice, how to optimize the spatial allocation of the warming effect of the buildings and the cooling effect of these open spaces, in order to jointly deal with the climate problems in the process of urbanization, is worth more indepth exploration. For instance, the results of this study can be used to identify areas in which planting new trees and planning new buildings may most effectively mitigate UHI.

\section{Conclusion}

This study investigated the scale effect of 3D building morphology parameters (the number, area, height, volume, and the surface area of the buildings) in 31 provincial capitals of China city on the UHI effect. The results showed that the building area had the greatest influence on the relative UHI intensity in summer daytime, and the building height had the slightest influence on the relative UHI intensity. These effects were more significant in the spatial scale of $150 \mathrm{~m}-540 \mathrm{~m}$, and the impacts of the buildings on urban thermal environment showed a larger spatial scale in developed cities.

The research results are of great significance to improve the urban thermal environment through urban planning and urban renewal. Especially, it provides the theoretical basis and technical support for the planning decision-makers to control the total amount and the height of urban buildings within the appropriate spatial scale and to configure spatially the urban cold island infrastructure.

\section{Data Availability}

The Landsat- 8 data used to support this study were supplied by the NASA and USGS under license and it is not available. The building outline vector data were supplied by the Chinese Academy of Sciences under license and it is not available. Requests for access to these data should be done through contacting the NASA and USGS and the Resource and Environment Data Cloud Platform (http://www.resdc.cn/Default. aspx) of the Chinese Academy of Sciences, respectively.

\section{Conflicts of Interest}

The authors declare that there are no conflicts of interest regarding the publication of this paper.

\section{Acknowledgments}

This study was supported by the National Natural Science Foundation of China (Grant numbers: 41971389 and 41501472) and Strategic Priority Research Program of the Chinese Academy of Sciences (Grant number: XDA20010302).

\section{Supplementary Materials}

Supplementary materials Table 1: the information of the collected remote sensing images in each city. (Supplementary Materials)

\section{References}

[1] K. C. Seto, B. Guneralp, and L. R. Hutyra, "Global forecasts of urban expansion to 2030 and direct impacts on biodiversity and carbon pools," Proceedings of the National Academy of Sciences, vol. 109, no. 40, pp. 16083-16088, 2012.

[2] T. R. Oke, "The energetic basis of the urban heat island," Royal Meteorological Society, vol. 108, pp. 1-24, 1982.

[3] S. Peng, S. Piao, P. Ciais et al., "Surface urban heat island across 419 global big cities," Environmental Science \& Technology, vol. 46, no. 2, pp. 696-703, 2012.

[4] D. Zhou, S. Zhao, S. Liu, L. Zhang, and C. Zhu, "Surface urban heat island in Chin's 32 major cities: spatial patterns and drivers," Remote Sensing of Environment, vol. 152, pp. 51-61, 2014.

[5] Z. Qiao, C. Wu, D. Q. Zhao et al., "Determining the boundary and probability of surface urban heat island footprint based on a logistic model," Remote Sensing, vol. 11, no. 11, p. 1368, 2019.

[6] D. C. Zhou, J. F. Xiao, S. Bonafoni et al., "Satellite remote sensing of surface urban heat islands: progress, challenges, and perspectives," Remote Sensing, vol. 11, no. 1, 2019.

[7] X. Y. Zhang, M. A. Friedl, C. B. Schaaf, A. H. Strahler, and A. Schneider, "The footprint of urban climates on vegetation phenology," Geophysical Research Letters, vol. 31, no. 12, 2004.

[8] C. Cao, X. H. Lee, S. D. Liu et al., "Urban heat islands in China enhanced by haze pollution," Nature Communications, vol. 7, 2016.

[9] M. Santamouris, C. Cartalis, A. Synnefa, and D. Kolokotsa, "On the impact of urban heat island and global warming on the power demand and electricity consumption of buildings-a review," Energy and Buildings, vol. 98, pp. 119-124, 2015. 
[10] J. O’Loughlin, F. D. W. Witmer, A. M. Linke, A. Laing, A. Gettelman, and J. Dudhia, "Climate variability and conflict risk in East Africa, 1990-2009," Proceedings of the National Academy of Sciences, vol. 109, no. 45, pp. 18344-18349, 2012.

[11] United Nations Department of Economic Social Affairs Population Division, World Urbanization Prospects: The 2018 Revision, United Nations Publications, New York, NY, USA, 2018.

[12] Z. Qiao, G. Tian, and L. Xiao, "Diurnal and seasonal impacts of urbanization on the urban thermal environment: a case study of Beijing using MODIS data," ISPRS Journal of Photogrammetry and Remote Sensing, vol. 85, pp. 93-101, 2013.

[13] T. R. Oke, G. Mills, and J. A. Voogt, Urban Climates, Cambridge University Press, Cambridge, UK, 2017.

[14] K. Deilami, M. Kamruzzaman, and Y. Liu, "Urban heat island effect: a systematic review of spatio-temporal factors, data, methods, and mitigation measures," International Journal of Applied Earth Observation and Geoinformation, vol. 67, pp. 30-42, 2018.

[15] C. Berger, J. Rosentreter, M. Voltersen, C. Baumgart, C. Schmullius, and S. Hese, "Spatio-Temporal analysis of the relationship between 2D/3D urban site characteristics and land surface temperature," Remote Sensing of Environment, vol. 193, pp. 225-243, 2017.

[16] G. L. Feyisa, K. Dons, and H. Meilby, "Efficiency of parks in mitigating urban heat island effect: an example from Addis Ababa," Landscape and Urban Planning, vol. 123, pp. 87-95, 2014.

[17] E. A. Gage and D. J. Cooper, "Relationships between landscape pattern metrics, vertical structure and surface urban heat island formation in a Colorado suburb," Urban Ecosystems, vol. 20, no. 6, pp. 1229-1238, 2017.

[18] E. A. Gage and D. J. Cooper, "Urban forest structure and land cover composition effects on land surface temperature in a semi-arid suburban area," Urban Forestry \& Urban Greening, vol. 28, pp. 28-35, 2017.

[19] J. Yang, J. Su, J. Xia, C. Jin, X. Li, and Q. Ge, “The impact of spatial form of urban architecture on the urban thermal environment: a case study of the Zhongshan district, Dalian, China," IEEE Journal of Selected Topics in Applied Earth Observations and Remote Sensing, vol. 11, no. 8, pp. 27092716, 2018.

[20] J. Yang, S. H. Jin, X. M. Xiao et al., "Local climate zone ventilation and urban land surface temperatures: towards a performance-based and wind-sensitive planning proposal in megacities," Sustainable Cities and Society, vol. 47, pp. 1-11, 2019.

[21] Z. Qiao, X. Xu, F. Wu et al., "Urban ventilation network model: a case study of the core zone of capital function in Beijing metropolitan area," Journal of Cleaner Production, vol. 168, pp. 526-535, 2017.

[22] J. Li, C. Song, L. Cao, F. Zhu, X. Meng, and J. Wu, "Impacts of landscape structure on surface urban heat islands: a case study of Shanghai, China," Remote Sensing of Environment, vol. 115, no. 12, pp. 3249-3263, 2011.

[23] K. J. Bhang and S. S. Park, "Evaluation of the surface temperature variation with surface settings on the urban heat island in Seoul, Korea, using landsat-7 ETM+ and spot," IEEE Geoscience and Remote Sensing Letters, vol. 6, no. 4, pp. 708-712, 2009.

[24] C. Yin, M. Yuan, Y. Lu, Y. Huang, and Y. Liu, "Effects of urban form on the urban heat island effect based on spatial regression model," Science of the Total Environment, vol. 634, pp. 696-704, 2018.
[25] A. K. Nassar, G. A. Blackburn, and J. D. Whyatt, "Dynamics and controls of urban heat sink and island phenomena in a desert city: development of a local climate zone scheme using remotely-sensed inputs," International Journal of Applied Earth Observation and Geoinformation, vol. 51, pp. 76-90, 2016.

[26] M. Scarano and J. A. Sobrino, "On the relationship between the sky view factor and the land surface temperature derived by landsat- 8 images in Bari, Italy," International Journal of Remote Sensing, vol. 36, no. 19-20, pp. 4820-4835, 2015.

[27] I. D. Stewart and T. R. Oke, "Local climate zones for urban temperature studies," Bulletin of the American Meteorological Society, vol. 93, no. 12, pp. 1879-1900, 2012.

[28] A. D. Guo, J. Yang, W. Sun et al., "Impact of urban morphology and landscape characteristics on spatiotemporal heterogeneity of land surface temperature," Sustainable Cities and Society, vol. 63, Article ID 102443, 2020.

[29] J. Yang, Y. Wang, C. Xiu, X. Xiao, and C. Jin, "Optimizing local climate zones to mitigate urban heat island effect in human settlements," Journal of Cleaner Production, vol. 275, Article ID 123767, 2020.

[30] J. Yang, Y. X. Zhan, X. M. Xiao, J. H. Xia, W. Sun, and X. M. Li, "Investigating the diversity of land surface temperature characteristics in different scale cities based on local climate zones," Urban Climate, vol. 34, Article ID 100700, 2020.

[31] C. D. Ziter, E. J. Pedersen, C. J. Kucharik, and M. G. Turner, "Scale-dependent interactions between tree canopy cover and impervious surfaces reduce daytime urban heat during summer," Proceedings of the National Academy of Sciences, vol. 116, no. 15, pp. 7575-7580, 2019.

[32] Z. Qiao, L. Liu, Y. Qin, X. Xu, B. Wang, and Z. Liu, "The impact of urban renewal on land surface temperature changes: a case study in the main city of Guangzhou, China," Remote Sensing, vol. 12, no. 5, p. 794, 2020.

[33] K. Zanter, "Landsat8 (L8) data users handbook," 2020, https:// www.usgs.gov/core-science-systems/nli/landsat/landsat-8data-users-handbook.

[34] A. A. Van de Griend and M. Owe, "On the relationship between thermal emissivity and the normalized difference vegetation index for natural surfaces," International Journal of Remote Sensing, vol. 14, no. 6, pp. 1119-1131, 1993.

[35] A. Black and H. Stephen, "Relating temperature trends to the normalized difference vegetation index in las vegas," GIScience \& Remote Sensing, vol. 51, no. 4, pp. 468-482, 2014.

[36] O. Rozenstein, Z. Qin, Y. Derimian, and A. Karnieli, "Derivation of land surface temperature for landsat-8 TIRS using a split window algorithm," Sensors, vol. 14, no. 4, pp. 5768-5780, 2014.

[37] Z. Qin, G. Dall'Olmo, A. Karnieli, and P. Berliner, "Derivation of split window algorithm and its sensitivity analysis for retrieving land surface temperature from NOAA-advanced very high resolution radiometer data," Journal of Geophysical Research: Atmospheres, vol. 106, no. D19, pp. 22655-22670, 2001.

[38] Y. Zhang, P. Jiang, H. Y. Zhang, and P. Cheng, "Study on urban heat island intensity level identification based on an improved restricted boltzmann machine," International Journal of Environmental Research and Public Health, vol. 186, no. 2, 2018.

[39] S. M. Sun and C. Y. Lu, "Study on monitoring intensity of urban heat island and taking it as an indicator for urban ecosystem by remote sensing," Journal of Xiamen University (Natural Science), vol. 41, no. 1, pp. 66-70, 2002, In Chinese.

[40] C. Yuan and L. Chen, "Mitigating urban heat island effects of high density cities: a study at Hong Kong," Architectural Science Review, vol. 54, no. 4, pp. 305-315, 2010, In Chinese. 